\title{
EDITORIAL
}

\section{Type I IFNs and $M$. bovis BCG vaccine: The sword to target ger- minal centers and unlock humoral immunity in leprosy}

\author{
Gloria G Guerrero $M^{*}$ \\ Unidad Académica de Ciencias Biológicas. Universidad Autonoma de Zacatecas. Zacatecas, Zac. Mexico
}

\begin{abstract}
Leprosy is still a serious human health problem in developing countries. Environmental and genetic factors are playing a key role in the chronic course of the disease, resistance versus susceptibility. Multidrug treatment is not effective for all infected individuals. "Cured" individuals mostly show relapses of neurological disorders as the same as not cured can present physical and deformed constraints. The unsolved matter in leprosy is that the clinical spectrum depends on the host immune response.and thus, the outcome of the immune response. In the present review we intend to describe some aspects of the immunotherapy, based on type I IFNs and $M$. bovis BCG vaccine like a sword strategy to target germinal centers, either for its generation or for its enhancement and thus, throughout key signals delivered by follicular CD4+ T cells and controlled by follicular regulatory CD4+ T cells, B cell differentiation into plasmacytoid cells being highly promotes the induction of protective high affinity neutralizing antibodies to unlock humoral immunity towards M. leprae infected individuals.
\end{abstract}

Keywords: type I IFNs; M. bovis BCG; germinal centers; leprosy; humoral immunity

ARTICLE INFO

Received: January 11, 2019

Accepted: January 28, 2019

Available online: February 7, 2019

*CORRESPONDING AUTHOR

Gloria G Guerrero M, Unidad Académica

de Ciencias Biológicas. Universidad Au-

tonoma de Zacatecas. Zacatecas, Zac.

Mexico; gloriaguillermina@uaz.edu.mx;

gloguerrero9@gmail.com

\section{CITATION}

Guerrero GG. Type I IFNs and M. bovis BCG vaccine: The sword to target germinal centers and unlock humoral immunity in leprosy. Trends Immunother 2019; 3(1): 3-9.

doi: 10.24294/ti.v3.i1.1122

\section{COPYRIGHT}

Copyright ( $\odot 2019$ by author(s) and EnPress Publisher LLC. This work is licensed under the Creative Commons AttributionNonCommercial 4.0 International License (CC BY-NC 4.0). http://creativecommons. org/licenses/by/4.0/

\section{Introduction}

Hanses's infection disease (human leprosy) caused by Mycobacerium leprae is an ancient world infectious disease ${ }^{[1,2]}$ that still constitutes a serious threat to human beings in developing countries ${ }^{[1-3]}$. The multidrug treatments are not effective for all infected individuals. The genetic susceptibility to the disease as well as the environmental (poverty, geographic localization) plays a pivotal role in the development of the clinic manifestations and the outcome of the host immune response ${ }^{[4-7]}$. How and whether or no epigenetic factors influence the outcomes in a manner positive or negative, are issues that deserve further studies ${ }^{[8,9]}$. A major limitation that has dampened advances in the understanding of the immunopathogenesis of leprosy is that M. leprae could not be propagated in vitro. However, nowadays, cellular immunology of leprosy can be approximated in vitro throughout keratinocytes infection ${ }^{[10]}$ as well as human airway epithelial cells ${ }^{[11]}$. Moreover, tick cell lines constitute a step forward to a promising venue not only for large scale production of $\mathrm{M}$. leprae bacteria but for deep insight into the mechanism of pathogenici$\operatorname{ty}^{[12]}$.

M. leprae is an alcohol acid resistant intracellular bacilli pathogen ${ }^{[9,10]}$ comprised of at least 33 immunodominant antigens (genome scan) ${ }^{[12]}$, among them a 19 and 33Kda lipoproteins that interact through pattern pathogen receptors (PRRS), Toll-like receptors on innate cells (macrophages, dendritic cells) ${ }^{[13-15]}$ to trigger an acute pro-inflammatory state accompanied with TNF-alpha and IL-12 to connect with adaptive immune response (CMI) ${ }^{[16-19]}$. In leprosy either in humans (M. leprae) or in mice ( $M$. lepraemurium), the host-pathogen interaction leads to a spectrum of cellular immune and clinical phenotype ${ }^{[18-20]}$. As outlined in 
Figure 1, in one extreme of the spectrum, tuberculoid (TT/) on strong Th1 and Th17 immune response that limits the pathogen and dissemination. In the middle, two intermediate phenotypes, Borderline tuberculoid (BT) and Borderline (BB), a mix of a Th1 and Th2 cellular immune response. Moreover, TLR1, TLR2 enhanced expression in resistant infected patients (Tuberculoid Leprosy) and down regulation in susceptible infected $(T L L)^{[5,19]}$. What determines that each of these intermediae stages is polarized toward a predominant Th1 or Th2?. On the other end of the spectrum a lepromatous disease or borderline lepromatous leprosy with a Th2 type cellular immune response, and a regulatory $\mathrm{T}$ cellular component (CD4-FoxP3+) that can not limit the dissemination of the pathogen and is dominated by a state of anergy (Figure 1). Indeed, leprosy regulatory $\mathrm{T}$ or $\mathrm{B}$ cells leads to a immunosuppressive ambiance by IL-10, TGF-beta induction instead oof IL17 protective immune responses and avoid exacerbates helper follicular $\mathrm{T}$ cell as well as $\mathrm{B}$ cell proliferation ${ }^{[21-25]}$. The state of immunosuppression has been proposed to play a pivotal role in the immunopathogenesis and immunoregulation not only in human leprosy but also in auto- inmune diseases ${ }^{[25]}$. The role of regulatory T cells is to control or regulate functions in different sites, however, in some infectious diseases such as leprosy it is intriguing that $\mathrm{B}$ and $\mathrm{T}$ regulatory $\mathrm{T}$ and $\mathrm{B}$ cells instead of immunoregulate promotes a immunosuppressive state for the establishment of the pathogen. Therefore, it is tempting to think that in eradicated regions, the treatments promoted a strong Th1 and Th17 immune protective response while in endemic regions, infected individuals have a dysregulation of the immune response, and the induction of a state of anergy that hampers elimination of the pathogen. How the Th1 cellular protective immune response can be augmented against disiduos complex pathogens like M. leprae?. How to resolve this immunological challenge and moreover, how to modulate it in order to obtain more beneficial protective responses than damage to the host. One hypothesis that raises from different studies is that vaccines adjuvants against infected individuals might be directed germinal centers because these sites are programmed for high affinity neutralizing protective antibodies induction either for bacterial or virus infections ${ }^{[26,27]}$.

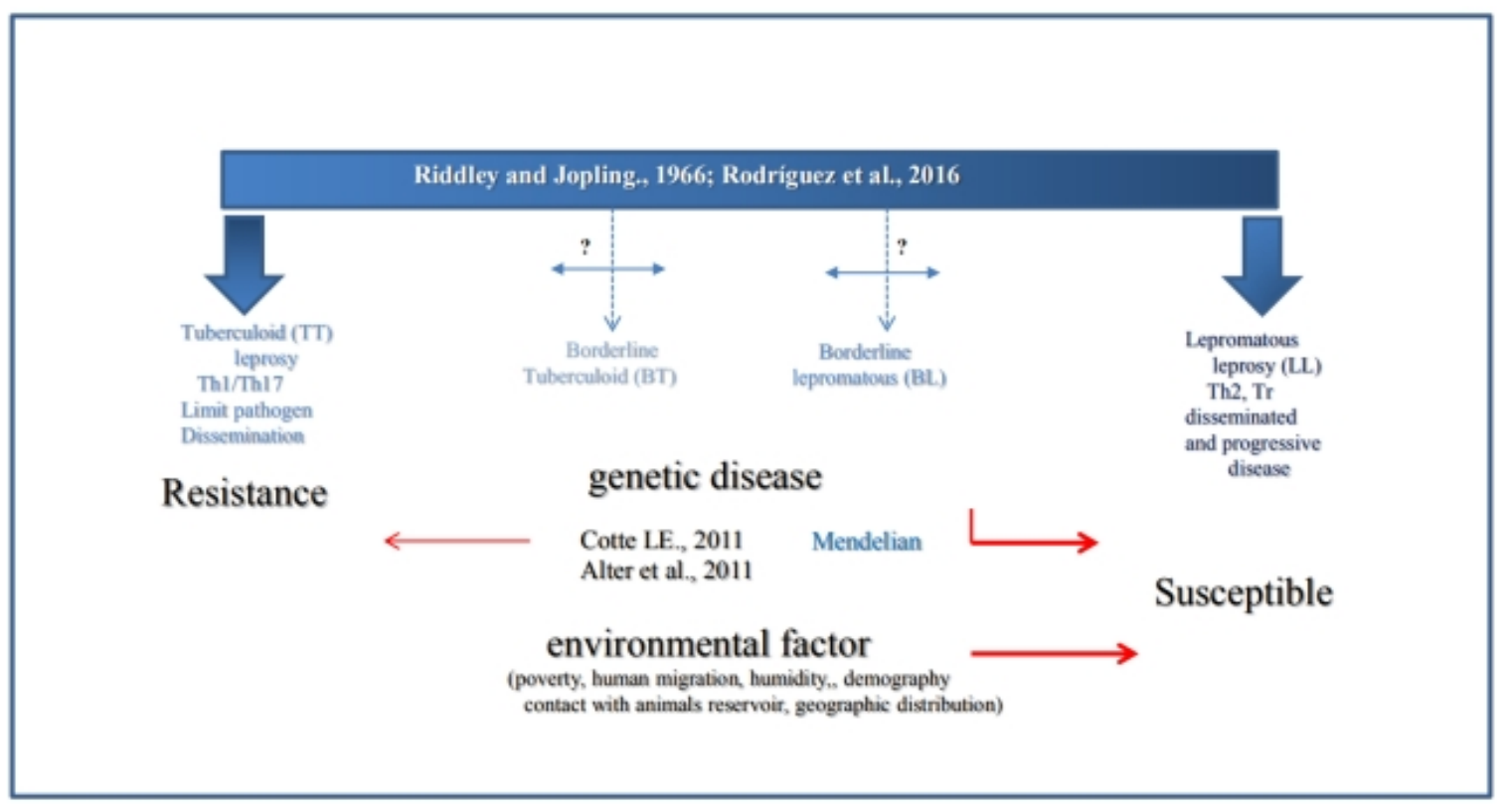

Figure 1. Outline of the clnical spectrum of the host immune response in leprosy.

Riddley and Jopling in 1966 defined Hanse's disease or leprosy,-caused by Mycobacterium leprae, -as a heterogenous infectious disease because it shows a wide spectrum of clinic manifestations. M. leprae is monomorphic, there are not divergent variability among strains, making more feasible to study and vaccine development. Howev- er, multidrug treatments are not effective for all infected individuals presumably because the environmental factors and genetic susceptibility to disease. 


\section{Germinal centers}

Germinal centers (GCs), are niches inside secondary lymphoid organs (lymph node, spleen cells), that arise from the center of primary B follicles, programmed for the antigen diversification through $B$ cell receptor (BCR) somatic hypermutation (SHM). This process can be simple for simple antigens (protein), few mutations are required, but in the case of a more complex pathogen (virus, bacteria), protective since high affinity antibodies require a much higher rate of mutations ${ }^{[26,27-29]}$. The program of the high antibody diversity generation is well ordered and not limited, includeing that follicular $\mathrm{T}$ cells provide key signals to $\mathrm{B}$ cells for plasmacytoid differentiation and memory B cells induction, clonal expansión, hypersomatic mutation, and class switch of class. The output of the GCs reactions depends on several factors, in between of the interaction of stromal follicular dendritic cells (FDCs), follicular B cells (FBC), follicular helper $\mathrm{T}$ cells $(\mathrm{Tfh})$, follicular regulatory $\mathrm{T}$ cells (TFr), foamy macrophages in $\mathrm{GCs}^{[30-34]}$. The understanding of all GCs reactions that occur in secondary lymphoid organs (SLOs) or in tertiary lymphoid organs (TLOs) is pivotal for the development of vaccine ${ }^{[35-37]}$. GC reaction or generation of GC is an ordered process that involves different innate and adaptive cell populations, and all the reactions should be carefully regulated in order for rare antigens to be able to mount a high quality number of antibodies ${ }^{[35,36]}$. In the light of our data and from the literature, we think that this potential adjuvant vaccine (induction of GCs in secondary lymphoid tissues) is promising since the hallmark of vaccination and development of human vaccines are the induction of neutralizing protective antibody responses that depends strongly on CD4+ T cell population and germinal centers (programmed for antibody diversity generation, clonal expansión, hypersomatic mutation, switch of class), and thus, to have enhanced affinity and maturation of the humoral response which could primarily boost IgG subclass antibodies magnitude ${ }^{[38,39]}$.

From the outside, the lifespan of GCs, can be modulated by adjuvants vaccines, by immunization protocols or strategies to long lastly Ag availability $^{[37,38]}$. To approximate this first in vivo and then in vitro, we had used $M$. lepraemurium mice infection whose primarily and general clinic manifestation is skin lesión and peripheral nerve damage $\mathrm{e}^{[16]}$. In murine leprosy at early times of infection, Th1 type cellular immune response limits the pathogen dissemination reflecting the tuberculoid phenotype. But weeks later, there is a dysregulation of either cellular and humoral immune response that last with a state of anergy giving rise to lepromatous phenotype ${ }^{[40-42]}$.

\section{Type I Interferons}

In agreement with already data from the literature ${ }^{[43-47]}$, we had found that the sole administration of type I IFNs exacerbate $M$. lepraemurium and/or M. tuberculosis infection. Firstly, skin lesión was not developed ${ }^{[48]}$ while in the second a reduction of the bacterial load was observed ${ }^{[49]}$. Interestingly, we found in either infection model as well as from in vitro experiments ( $M$. tuberculosis) that the treatment based on type I IFNS plus BCG vaccine promote strong Th1 cellular immune response and protection against $M$. tuberculosis infection ${ }^{[49]}$, whereas in murine leprosy type I IFNs induced protection correlated with a high induction of iNOS induction $^{[48]}$, The mechanism of this induced protection in either model are studies that are currently addressed. As part of an approximantion of the anergy observed at the level of the celular and in the humoral immune response, we asked how adjuvants such as type I IFNs could have an influence on GCs processes in secondary or tertiary lymphoid organs?

Type I IFNs are induced at a very low steady level in the absence of bacterial or viral infection ${ }^{[43-}$ ${ }^{45]}$ (Figure 2). However, after infection (bacterial or viral) or immunization, type I IFNs signalization on innate cells (dendritic cells, DCs) thorough IFN receptor (IFNR) (Figure 2) induce the expression in mesenteric lymph node of CXCR5+ follicular $\mathrm{T}$ helper, cells to support plasmacytoid B cell differentiation into plasmablasts and plasma cells that secrete high affinity switched Abs as well as the generation of memory B cells, ${ }^{[22,23]}$ leading thus to an enhanced cross protective immune response. CXCR5+ expression also enables B cells to be recruited to the lung and thus, initiate formation of functional GCs (Figure 2). Furthermore, type I IFNs after viral infection can induce CXCL-13 expression in lung fibroblast, conducting thus, to CXCR5+ dependent recruitment of $B$ cells, leading to initiation of ectopic GCs formation ${ }^{[31,32]}$. This supports a role of type I IFNs as a natural adjuvant of SLO or TLO lung remodeling ${ }^{[31,32]}$. Is this a direct evidence that type I IFNs induce more efficient germinal centers in mice. It has been described that GCs enhancement and therefore humoral immune responses potency can be modulated throughout extended antigen availability favored by vaccine adjuvant and of the 
immunization strategies ${ }^{[27]}$. The mechanism of this had been proposed to occur precisely as stated above through improving $\mathrm{T}$ follicular helper (Tfh) cell generation which will lead to enhanced affinity maturation and enhanced development of $\mathrm{B}$ ell memory. From our own findings, successive IFNalpha boosting of $M$. bovis BCG vaccinated mice, increased GCs average in SLO that correlated with induced protection in intradermal infected mice (lack of skin lesion) (Guerrero et al., unpublished results). How type I IFNs natural action in infected $M$. leprae individuals is proposed to initiate; as outlined by ${ }^{[46]}$, toll like receptors (TLR2-/1), Interferon 1 and 2 receptors (IFNR); intracellular nucleotide binding oligomerization domain containging (NOD2)NOD-receptors, trigger a series of pathways which final commitment is the shut off or at least downregulation of pro-inflammatory response (macrophage microbicidal mechanisms, oxide nitric synthetase (iNOS, Il1- $\beta$, IL-6, TNF- $\alpha$, IL-12, IFN- $\gamma$ ) against versus antiinflammatory respose ( $M$. leprae or M.tuberculosis infection) represented by a suppressive state leaded by IL10; the stimulator of interferón genes (STING); interferón regulatory factor 3(IRF3, cGAS (cGMP-AMP syntahse); [2'-5'oligoadenylate synthetase](OASL). But also there is a change in the energy supply through glucose uptake, pentose pathway that generates, nicotinamide adenine dinucleootide phosphate (NADPH), lipids metabolism (arachidonic acids, eicosanoids, leukotrienes), as well as an increase in mitocondrial activity that serve to the own pathogens purposes, a switch off in the metabolism to redirect carbon(s) source for nutrients survival inside lipid bodies) that it has been observed in the skin lesion of M. leprae infected patients ${ }^{[46,47]}$ (Figure 2). Autophagy and a pro-inflammatory response are activated upon NOD2 recogniton of mycobacterial DNA, that initiate a leucine-rich-repeat kinase 2 (LRRK2)dependent response followed by host's recruitment of ubiquitin chains, exemplified by E3-ubiquitin ligase parkin (PARK2) to target mycobacteria to autophagic degradation (Figure 2). The evasion mechanism used by is the early secreted system (ESTX-1) mediate pore formation in the autophagosomal membrane to equalize autophagosomal and cytosol content. This, eventually trigger type I IFNs response and autophagy and downregulation of IL1$\beta$. Interestingly, as depicted in Figure 2, the sword represented with $M$. bovis BCG vaccine plus IFNalpha propose that this strategy might be redirecting type I IFNs adjuvant properties toward an increase in the antigen availability favored at the same time through an increase in the CXCR5+CD4+T cell generation and further CXCL13+ expression in SLO or TLO follicular B and T cells, key players to unlock humoral immuner responses in GC.

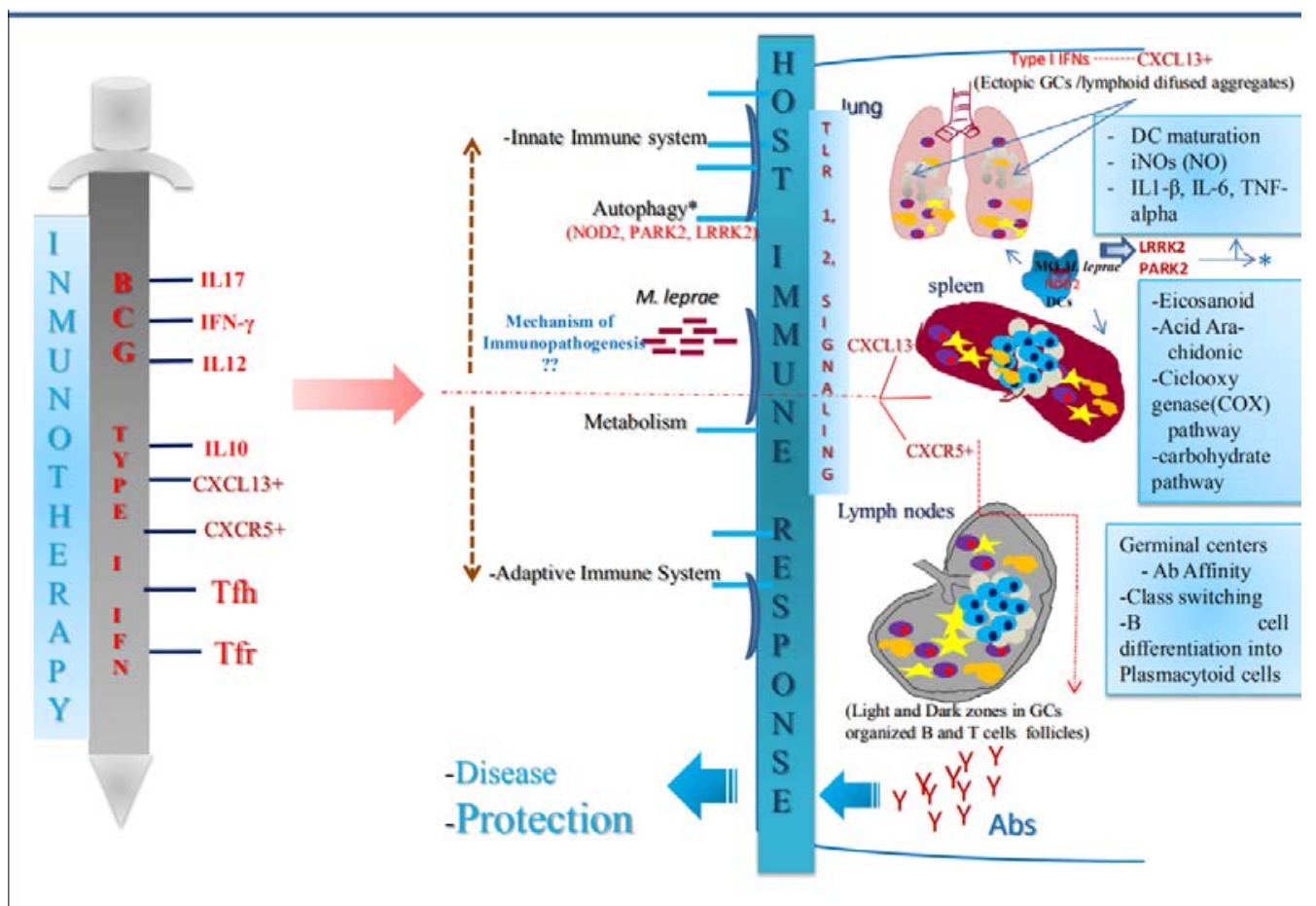

Figure 2. Scheme of type I IFNs and M. bovis BCG vaccine immunotherapy as a sword in the host immune response in leprosy. 
In the $M$. leprae infection, the outcome of the immune response depends of the effectiveness in the generation of key innate and cellular host immune system at systemic and mucosal tissues. type I IFNs adjuvant action in synergy with $M$. bovis BCG vaccine faced host immune response, such as autophagy, metabolism upon $M$. leprae infection. Intracellular receptors such as nucleotide binding oligomerization domain containing 2 (NOD2) upon recognition of muramyl-dipeptides of $M$. leprae (first interaction with Toll-like receptors, TLR2/1) can initiate a leucine-rich-repeat-kinase 2(LRRK2)dependent pro-inflammatory response as well as autophagy. While targeting of $M$. leprae/M. tuberculosis for autophagic degradation involves recruitment of the host ubiquitin system that depends on an E3-ubiquitin-ligase or parkin encoded by the gene PARK2, associated with leprosy susceptibility. Once the pathogen enter and switch the metabolism of glycolysis, lipids and cholesterol for its own purposes, it is highly possible that type I IFNs robust adjuvanticity plus $M$. bovis BCG redirect its action to unlock humoral immune responses throughtout enhancement of CXCL13+ (lung fibroblast) and CXCR5+ in lymph node to enhance follicular $\mathrm{T}$ helper cells (Tfh) cells that are primordial to deliver key signals for B cell differentiation in GCs to plasmacytoid cells for the production of high affinity neutralyzing antibodies. These processes are modulated by follicular regulatory $\mathrm{T}$ cells (Tfr). Moreover, it is tempting to think that the sword of the proposed immunotherapy pin-point the antigen availability potentially favored by the successive immunization strategy of IFN-alpha to $M$. bovis BCG vaccinated mice.

\section{Conclusions}

In the immunobiology of leprosy (M. leprae/M. lepraemurium), most of the studies have focused to search for therapeutic targets etiher from genome wide studies (GWS) or from microarray expression profiles which have pin point several genes of susceptibility or resistance as well as potential biomarkers of the different interrelated pathways such type I IFNs, autophagy and metabolism. But still remains unsolved others aspects in the biology of the disease. From our own findigns, successive IFN-alpha boosting of $M$. bovis BCG vaccinated mice, increased GCs average in SLO that correlated with induced protection (lack of skin lesión development) (Guerrero et al., unpublished results). As depicted in figure 2, the sword comprised of $M$. bovis BCG vaccine plus IFN-alpha propose that this strategy might be redirecting type I IFNs adjuvant properites toward an increase in the antigen availability favored at the same time through an increase in the CXCR5+CD4+T cell generation and further CXCL13+ expression in SLO or TLO follicular B and $\mathrm{T}$ cells, key players to unlock humoral immuner responses in GCs.

\section{Acknowledgements}

GGGM is grateful to SNI-CONACYT (20162019) and SEP (Perfil Prodep program) for financial support.

\section{References}

1. Global leprosy update. 2014. Need for early case detection. Wdy. Epdiemiol. Rec. 2015; 90: 461474

2. Lastoria JC, and MA Abreu. Leprosy review of the epidemiological clinical and etnopathogenic aspects-part 1. An Bras Dermatol. 2014; 89: 2015-218

3. Rodriguez JIA, Gresta IT, Noviello M de I, et al. Leprosy classification methods: a comparative study in a referral character in Brazil. Int. J. Infect. Dis. 2016; 45: 118-122

4. Cottle LE. Mendelina susceptibility to mycobacterial disease. Clin Genet. 2011: 79: 17-22

5. Alter A, Grant A, Abel L, et al. Leprosy as a genetic disease. Mamm Genome. 2011; 22: 19-31

6. Bochud PV, Hawn TR, Aderem. Cutting edge: a Toll-like receptor 2 polymorphism that is associated with lepromatous leprosy is unable to mediate mycobacterial signaling. J. Immunol. 2003; 120: 3451-354

7. Alcais A, Alter A, Antoni G, et al. Stepwise replication identifies a low-producing lymphotoxinalpha allele as a major risk factor for early-onset leprosy. Nat.Genet. 2007; 39: 517-522

8. Adams BL, Pena TM, Sharma R, et al. Insights from animal models on the immunogenetics of leprosy. Mem Inst. Oswaldo Cruz. 2012; 107:197-208

9. Adams LB, Scollard DM, Ray NA, et al. The study of Mycobcaterium leprae infection in interferon-gamma gene-disrupted mice as a model to explore the immunopathologic spectrum of leprosy. J. Infect Dis. 2002; 185: 81-88

10. Lyrio EC, Campos-Souza IC, Corrêa LC, et al. Interaction of Mycobacterium leprae with the HaCaT human keratinocyte cell line: new frontiers in the cellular immunology of leprosy. Exp Dermatol. 2015; 24:536-42. doi: 10.1111/exd.12714

11. Silva CA, Danelishvili L, McNamara M, et al. Interaction of Mycobacterium leprae with human 
airway epithelial cells: adherence, entry, survival, and identification of potential adhesins by surface proteome analysis. Infect Immun. 2013:2645-59. doi: 10.1128/IAI.00147-1

12. da Silva FJ, Souza ODA, Santos JP, et al. Ticks as a potential vectors of Mycobacterium leprae; Use of tick cell lines to culture the bacilli and generate transgenic strains. Plos Negl Trop Dis. 2019; $\quad$ 12: e0007001. doi.org/10.1371/journal.pntd.0007001

13. Riddley DS and Jopling WH. Classification of leprosy according to immunity. A five group system. Int. J. Lepr Other Mycobacteria Dis. 1966; 34: $255-273$

14. Oliveira RB, Ochoa MT, Sieling PA. Rea TH, et al. Expression of Toll-like receptor 2 on human Schwann cells: a mechanism of nerve damage in leprosy. Infect. Immun. 2003; 71: 1427-1433

15. Akira S, Tukeda K. Toll-like receptor signalling. Nat Rev. Immunol. 2004; 499-511

16. Robert L. Modlin. The innate immune response in leprosy. Curr. Opin Immunol. 2010; 22: 48-54

17. Silva-Mianda M, Rodriguez KW, Martinez CE, et al. Expression of cyclooxygenase-2, alpha 1-acidglycoprotein and inducible nitric oxide synthase in the developing lesions of murine leprosy. Int. J. Exp. Path. 2006; 87: 485-494

18. Lee DJ, Li H, Ochoa MT, et al. Integrated pathwayss for neutrophil recruitment and inflammation in leprosy. J. Infect Dis. 2010; 201: 558569.doi:1 10. 1086/650318

19. Fonseca AB, Simon MD, Cazzaniga RA, et al. The influence of innate and adaptative immune responses on the differential clinical outcomes of leprosy. Infect Dis Poverty. 2017; 6:5. doi: 10.1186/s40249-016-0229-3.

20. Tarique M, Saini C, Naz H, et al. Fate of T Cells and their Secretory Proteins During the Progression of Leprosy. Curr Protein Pept Sci. 2018;19:889-899. doi: 10.2174/1389203718666170829120729.

21. Amaral JJ, Antunes LC, de Macedo CS, et al. Metabonomics reveals drastic changes in antiinflammatory/pro-resolving polyunsaturated fatty acids-derived lipid mediators in leprosy disease. PLoS Negl Trop Dis. 2013; 7(8):e2381. doi: 10.1371/journal.pntd.0002381

22. Sadhu S, Khaitan BK, Joshi B, et al. Reciprocity between Regulatory T Cells and Th17 Cells: Relevance to Polarized Immunity in Leprosy. PLoS Negl Trop Dis. 2016 11;:e0004338. doi: 10.1371/journal.pntd.0004338. eCollection Victora DG and MC Nussenweig. Germinal centers. Annu Rev Immunol. 2012; 30: 429-437

23. Vinuesa CG, Linterman MA, Yu D, et al. Follicu- lat Helper T Cells. Annu. Rev. Immunol. 2016; 34: 335-368

24. Miles B, Connick E. Control of the Germinal Center by Follicular Regulatory T Cells During Infection. Front Immunol. 2018; 9:2704. doi: 10.3389/fimmu.2018.02704. eCollection 2018

25. Tarique M, Naz H, Kurra SV, et al. Interleukin10 Producing Regulatory B Cells Transformed $\mathrm{CD} 4^{+} \mathrm{CD} 25^{-}$Into Tregs and Enhanced Regulatory T Cells Function in Human Leprosy. Front Immunol. 2018 Jul 23;9:1636. doi: 10.3389/fimmu.2018.01636. eCollection 2018

26. Ding Y, Li J, Yang P, et al. Interleukin-21 promotes germinal center reaction by skewing the follicular regulatory $\mathrm{T}$ cell to follicular helper $\mathrm{T}$ cell balance in autoimmune BXD2 mice.. Arthritis Rheumatol. 2014; 66:2601-2612. doi: 10.1002/art.38735

27. Victora DG and MC Nussenweig. Germinal centers. Annu. Rev Immunol. 2012; 30: 429-437

28. Carelli MK and SH Crotty. Germinal center enhancement by extended antigen availability. Current Opin. Immunol. 2017; 47: 64-69

29. Litzler LC, Zahn A, Meli AP, et al. PRMT5 is essential for B cell development and germinal center dynamics. Nat Commun. 2019; 10:22. doi: 10.1038/s41467-018-07884-6

30. Xie MM, Dent AL. Unexpected Help: Follicular Regulatory T Cells in the Germinal Center. Front Immunol. 2018 Jul 2;9:1536. doi: 10.3389/fimmu.2018.01536

31. Bannard O, Cyster JG. Germinal centers: programmed for affinity maturation and antibody diversification. Curr Opin Immunol. 2017; 45:21-30. doi: 10.1016/j.coi.2016.12.004

32. Cucak H, Ydid U, Reizis B,et al. Type I Interferon signaling in Dendritic cells stimulates the development of Lymph Node-Resident $\mathrm{T}$ folicular helper cells. 2009. Immunity. 31: 491-501.

33. Denton EA, Innocentin S, Carr E, et al. Type IFNs induces CXCL13 to support ectopic germinal center formation. JEM. 2019. doi. Org. 10.1084/jem.20181216

34. Chen J, Li N, Yin Y, et al. Methyltransferase Nsd2 Ensures Germinal Center Selection by Promoting Adhesive Interactions between B Cells and Follicular Dendritic Cells. Cell Rep. 2018; 25:3393-3404.e6.

doi:

10.1016/j.celrep.2018.11.096

35. Soni C, Wong EB, Domeier PP, et al. B cellintrinsic TLR7 signaling is essential for the development of spontaneous germinal centers. J Immunol. 2014;193:4400-4414. doi: 10.4049/jimmunol.1401720. 
36. Peng C, Hu Q, Yang F, et al. BCL6-Mediated Silencing of PD-1 Ligands in Germinal Center B Cells Maintains Follicular T Cell Population. J Immunol. 2018; pii: ji1800876. doi: 10.4049/jimmunol.1800876

37. Basso K, Dalla-Favera R. BCL6: master regulator of germinal center reaction and key oncogene in B cell lymphomagenesis. Adv Immunol. 2010;105:193-210. doi: 10.1016/S00652776(10)05007-8

38. Arguni E, Arima M, Tsuruoka N, et al. JunD/AP1 and STAT3 are the major enhancer molecules for high Bcl6 expression in germinal center B cells. Int Immunol. 2006; 18:1079-89.

39. Le Bon A, Schiavoni G, D’Agostino G, et al. Type I interferons potently enhance humoral immunity and can promote isotype switching by stimulating dendritic cells in vivo. Immunity. 2001; 14: 461-470

40. Cooper AM, Adams LB, Dalton DK, et al. IFNgamma and NO in mycobacterial disease: new job for old hands. Trends Microbiol. 2002; 10: 221226

41. O'Garra A, Redford SP, McNab WF, et al. The immune response in Tuberculosis. Annu. Rev Immunol. 2013; 31: 475-527

42. Umemura M, Yahagi A, Hamada S, et al. IL-17mediated regulation of innate and acquired immune response against pulmonary Mycobacterium bovis Bacille Calmette-Güerin Infection. J
Immunol. 2007;178:3786-3796

43. Tovey MG, Lallemand CH, Meritet JF, et al. Adjuvant activity of interferon alpha: mechanism(s) of action. Vaccine 2006; 24: S46-S47

44. Prchal M, Pilz A, Simma O, et al, Müller M, Decker T. Type I Interferon as mediators of immune adjuvants for $\mathrm{T}$ and $\mathrm{B}$ cell dependent acquired immunity. Vaccine. 2009; 275: G17-G20

45. Hervas-Stubbs. Effects of IFN-alpha as a signal-3 cytokine on human naïve and antigen-experienced CD8+T cells. Eur.J. Immunol. 2010; 40: 33893402

46. Toledo Pinto TG, Batista-Silva LR, Medeiros RCA, et al. Autophagy and Host Metabolism in Leprosy. Front Immunol. 2018 Apr 23;9:806. doi: 10.3389/fimmu.2018.00806. eCollection 2018

47. Deretic V. Autophagy in infection. Curr. Opin. Cell Biol. 2010; 22: 252-262

48. Guerrero GG, Rangel-Moreno JJ, Islas-Trujillo S, et al. Successive Intramuscular boosting with IFN-alpha protects Mycobacterium bovis BCGvaccinated mice against $M$. bovismurium infection. BioMed Research International. 2015; doi: 10.1155/2015/414027

49. Rivas-Santiago C and Guerrero GG. Boosting of Mycobacterium bovis Bacillus Calmette GüerinVaccinated mice promoted Th1 type cytokines and protection against $M$. tuberculosis infection. BioMed Research International. 2017; doi:10.1155/2017/8796760 